\section{TUMOR HYPOXIA DRIVES SUPPRESSOR FUNCTION IN EXHAUSTED T CELLS LIMITING ANTITUMOR IMMUNITY}

${ }^{1}$ Paolo Vignali*, ${ }^{1}$ Kristin DePeaux, ${ }^{1}$ Mclane Watson, ${ }^{2}$ Nicole Scharping, ${ }^{1}$ Ashley Menk, ${ }^{1}$ Greg Delgoffe. 'University of Pittsburgh, Pittsburgh, PA, United States; '2University of California San Diego, La Jolla, CA, United States

Background While CD8 + cytotoxic T cells are clearly critical for identification and elimination of cancer cells, factors concentrated within the tumor microenvironment drive altered differentiation of these cells to a hypofunctional, short-lived state termed $\mathrm{T}$ cell exhaustion ${ }^{1}$ (figure 1a). Exhaustion is a progressive lineage, and it is now clear that terminally exhausted $\mathrm{T}$ (tTexh) cells are not the targets of checkpoint blockade immunotherapy but may serve as factors that limit immunotherapeutic efficacy. ${ }^{2-6}$ Compared directly, tumor-infiltrating CD8 + tTexh cells bear notable phenotypic similarity to CD4+Foxp3 + regulatory $\mathrm{T}$ (Treg) cells in expression of immunosuppressive molecules suggesting beyond loss of effector function, tTexh cells may be directly anti-functional and constrain tumor-specific immunity. Thus, we hypothesize that tTexh cells potentiate the suppressive microenvironment of solid tumor and that strategies to limit their generation or reprogram their immunosuppressive nature will improve control of tumor progression.

Methods T cell populations were isolated from murine tumor lines, B16-F10 melanoma, Ptenflox/floxBrafLSL.V600ETyr2Cre. ERT2-derived Clone 24 melanoma, MEER head and neck carcinoma, and MC38 adenocarcinoma. T cell-specific CD39 (Entpd1) deletion was accomplished by crossing Entpd1flox/ flox mice to Cd4Cre or E8iGFP-Cre-ERT2. Enforced expression of CD39 in effector T cells was attained by murine retroviral vector delivery. Tumor hypoxia was alleviated by CRISPR-Cas9-directed deletion of mitochondrial genes in B16F10 or by treatment with axitinib or metformin.

Results When sorted directly from tumor, CD8+PD-1hiTim-3 + tTexh cells, but not progenitor PD-1intTim-3- pTexh cells, induce marked suppression of $\mathrm{T}$ cell effector responses, comparable to CD4+Foxp3+ Treg cells from the same environment (figure $1 \mathrm{~b}-\mathrm{c}$ ). The ectonucleotidase, CD39, increases as cells progressively differentiate and is associated with terminal exhaustion. ${ }^{7} \quad 8$ CD8 $+\mathrm{T}$ cell-restricted deletion of CD39 restricts regulatory functions of tTexh cells (figure $1 \mathrm{~b}$ ), improving tumor control and augmenting response to checkpoint blockade (figure 1d). CD39 expression correlates with hypoxia exposure and tTexh cells sorted from tumors engineered to be less hypoxic or treated with hypoxia-mitigating agents displayed a significant loss of suppressive capacity. Our data suggest that tumor hypoxia enforces Hif1a-dependent expression of CD39 which depletes extracellular ATP, supports adenosine generation, and limits therapeutic efficacy.

Conclusions Our data support a model that as CD8 $+\mathrm{T}$ cells progress to terminal exhaustion, hypoxia exposure enforces the upregulation of CD39, providing tTexh cells a mechanism to suppress proinflammatory processes and promote tumor progression. These findings suggest tTexh cells are not solely dysfunctional but rather are deleterious to antitumor immunity and may need to be drastically reprogrammed or depleted to improve patient outcomes.
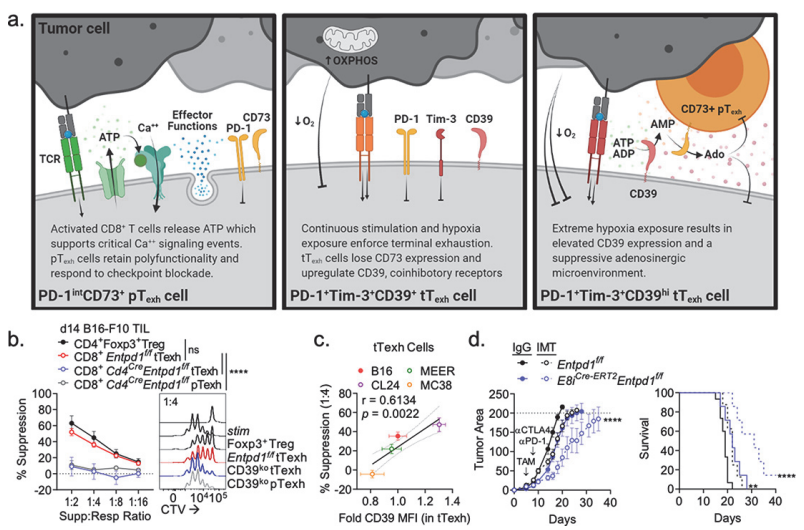

Abstract 679 Figure 1 (a) Schematic depicting differentiation of CD8 $+T$ cells to terminal exhaustion in cancer and subsequent suppression of local immune responses by expression of ectonucleosidase, CD39; (b) When assayed directly ex vivo, CD8+ terminally exhausted T (tTexh) cells, but not progenitor exhausted T (pTexh) cells, suppress effector functions as effectively as CD4+Foxp3+ Treg isolated from the same environment. Deletion of CD39 alleviates tTexh-mediated suppression; (c) CD8+ T cell suppression correlates with expression of CD39 on tTexh from various tumor models. (d) CD8+ T cell-specific deletion of CD39 slows tumor growth and improves immune response to checkpoint blockade-resistant tumors. Data are pooled from $\geq 3$ experiments. Statistics are two-way ANOVA with multiple comparisons or Pearson correlation. ${ }^{*} p<0.05,{ }^{* *} p<0.01,{ }^{* * *} p<0.001,{ }^{* * * *} p<0.0001$.

\section{REFERENCES}

1. Blank CU, et al. Defining "T cell exhaustion". Nat Rev Immunol 2019;19:665674.

2. Miller BC, et al. Subsets of exhausted CD8+ T cells differentially mediate tumor control and respond to checkpoint blockade. Nat Immunol 2019;20:326-336.

3. Blackburn SD, et al. Selective expansion of a subset of exhausted CD8 T cells by alphaPD-L1 blockade. Proc Natl Acad Sci USA 2008;105:15016-15021.

4. Sade-Feldman $M$, et al. Defining $T$ Cell states associated with response to checkpoint immunotherapy in Melanoma. Cell 2018;175:998-1013.e20.

5. Im SJ, et al. Defining CD8+ T cells that provide the proliferative burst after PD-1 therapy. Nature 2016;537:417-421.

6. Siddiqui I, et al. Intratumoral TCf1+PD-1+CD8+ T Cells with stem-like properties promote tumor control in response to vaccination and checkpoint blockade immunotherapy. Immunity 2019;50:195-211.e10.

7. Canale FP, et al. CD39 expression defines cell exhaustion in tumor-infiltrating CD8+ T Cells. Cancer Res 2018;78:115-128.

8 Gupta PK, et al. CD39 expression identifies terminally exhausted CD8+ T cells. PLOS Pathog 2015;11:e1005177.

http://dx.doi.org/10.1136/jitc-2021-SITC2021.679 